\title{
Determinación de la Presión Promedia de Yacimiento en Pruebas Multirata Aplicando la Tiab's Direct Synthesis Technique (TDST)*
}

\author{
Oscar Eduardo Ibagón Morera " \\ Harold Ernesto Silva Buitrago".
}

\section{Resumen}

La presión promedia de yacimiento, es un parámetro requerido en casi todos los estudios de ingeniería de yacimientos y de producción, al igual que en la mayoria de los trabajos de intervención de pozo. También, juega un papel crítico en la evaluación de campos, tamańo de pozos y diserío de los equipos y facilidades de superficie.

La determinación de la presión promedia se realiza por medio de lainterpretación de la información proveniente de pruebas de restauración de presión y pruebas bi-flujo o de dos ratas, las cuales presentan desventajas económicas en el caso de una prueba de restauración debido al clerre del pozo durante el tiempo de prueba y desventajas a nivel operacional en el caso de una prueba biflujo debido a la dificultad de mantener las dos ratas de flujo constantes durante la prueba. Por tal razón, surgió la idea de desarrollar una solución analítica que permitiera la determinación de la presión promedia de yacimiento en pruebas multirata, pruebas en las que la rata de flujo varía continuamente durante el tiempo de prueba y que no presentan las desventajas anteriormente senaladas.

En este articulo se presenta por primera vez una metodologia nueva, fácll y práctica para la determinación de la presión promedia de yacimiento en pruebas multirata durante estado pseudoestable para pozos verticales, localizados en regiones de drenajes cerrados. El método de interpretación de pruebas de presión denominado como la Tiab's Direct Synthesis Technique (TDST), asi como sus principios matemáticos, fueron utilizados para el desarrollo de la solución analifica aqui propuesta. Esta técnica usa una nueva ecuación analitica, la cual a su vez usa un único punto de presión y derivada de presión correspondiente al régimen de flujo pseudoestable, evitando el uso de cartas correctivas y curvas tipo. Para comprobar la eficacia de la soluclón propuesta, varios casos reales de campo y ejemplos simulados fueron analizados y resueltos, obteniendo valores bastante aceptables en comparación con los valores de presión promedia de yacimiento obtenidos con otros métodos y pruebas.

* Fneddy Humberb Escobar M. Dinector proyscto de grado. Ph.D. Ingenienía de Petrolleos Vicenector de huestigación y Proyec ción Social. Universidad Surcolombiana. fescobarousco. educo

*" Ingenisuo de Petrólicos. Universided Surcolombiana.

**t Ingeniaro de Patróleos. Universided Sumcolombiana. 
Palabras clave: Tiab's Direct Synthesis Technique (TDST), régimen de flujo pseudoestable, yacimiento cerrado, presión promedia de yacimiento.

\section{Abstract}

Average reservoir pressure is a required parameter in almost all reservoir and production engineering studies, the same as the majority of well intervention jobs. It also plays critical role in field appraisal, well sizing and equipment and surface facilities design.

Estimating of average reservoir pressure is done through interpretation from buildup, and two-rate tests which have disadvantages such as economical disadvantages for buildup tests due to well shut in during entire test and operational disadvantages for two-rate tests because it's really difficult to maintain constant the flowrates during the test. Because of this situation, the idea of develop an analytic solution which allows estimating the average reservoir pressure in multirate tests was generated. In these tests, the flowrate changing continuously during all test and they don't have the disadvantages above mentioned.

In this work, a new, easy and practical methodology is presented for first time, estimating average reservoir pressure in multirate tests during pseudosteady-state flow period for vertical wells located inside closed drainage regions. To develop the new analytic solution, the pressure transient testing method, referred to as Tiab's Direct Synthesis Technique (TDST) and its mathematical basis were used. This technique employs a new analytical equation which uses a single pressure point and the value of the pressure derivative corresponding to the time pseudosteady state period eliminating the use of correction charts and type-curve matching.

Several real field and simulated examples were analyzed and solved with the solution, in order to verify its effectiveness. The average reservoir pressure values were determined using the solution proposed in this work, show very good agreement with those estimated by other techniques and tests.

Keywords: Tiab's Direct Synthesis Technique (TDST), pseudosteady state regime, bounder reservoir, average reservoir pressure.

\section{Introducción}

L as pruebas de presión son de gran importancia en la industria petrolera, debido a que gracias a ellasse determinan caracteristicas y parámetros fundamentales del yacimiento, los cuales se tienen en cuenta en todas las fases de producción que tenga en particular el pozo y en general el yacimiento de estudio.

Las propiedades y parámetros obtenidos por lo general en las pruebas de presión son, almacenamiento, factor de dańo, permeabilidad, fronteras, límites del yacimiento y presión promedia de yacimiento, entre otros. Este último parámetro, la presión promedia de yacimiento, es de vital importancia ya que tiene gran incidencia en el comportamiento del yacimiento en cualquier etapa de su vida: recuperación primaria, secundaria y proyectos de mantenimiento de presión. La presión promediadeyacimientoseusaencaracterización de yacimientos, para calcular petróleo original, efectuar monitoreo del yacimiento mediante balance de materia, estimar indices de productividad y predecir el comportamiento y recobro final de un yacimiento.

Las pruebas de presión usadas para la determinación de la presión promedia de yacimiento, presentan algunas desventajas técnicas y especialmente económicas, las cuales en la mayoria de los casos las vuelven imprácticas. Las pruebas multirata nopresentan tales desventajas, estas tienen la gran ventaja de de dar datos de transientes de presión a medida que la producción de fluidos continua; además minimizan los cambios en el almacenamiento, disminuyen los efectos de segregación y 
redistribución de fases y remueven los efectos negativos de las fluctuaciones en la rata de flujo.

Aprovechando las ventajas que ofrecen las pruebas multiratay teniendo en cuentaque hasta la fecha no existía una solución o expresión para la determinación de la presión promedia de yacimiento con la información proveniente de este tipo de pruebas, se desarrolló una solución analitica para determinar este importante parámetro, mediante la aplicación de la Tiab's Direct Synthesis Technique (TDST), técnica que es considerada como una de las más prácticas y modernas para abordar lainformación obtenida de una prueba de presión. El presente articulo, muestra el desarrollo de la solución propuesta y los resultados obtenidos al aplicarla.

\section{Descripción del proyecto}

El proyecto en primera instancia, describe de forma general, las pruebas de transiente de presión y los conceptos relacionados con el cálculo de la presión promedia de yacimiento. En segunda instancia, son mostrados los principios matemáticos de las pruebas multirata y la aplicación de la Tiab's Direct Synthesis technique (TDST) a las mismas. Por medio de lasecuacionesquedescriben el comportamiento de la presión en las pruebas multirata, el estado de régimen de flujo pseudoestable y la ecuación que define la presión promedia de yacimiento, se generan las soluciones propuestas para la determinación de la presión promedia de yacimiento aplicando la TDST. Por último, se realiza la respectiva validación de las soluciones propuestas mediante casos reales de campo y ejemplos simulados, resultados bastante aceptables comparados con los obtenidos aplicando otros métodos.

\section{Metodología}

Un método de estudio sistemático, analítico y descriptivo fue utilizado para conseguir el objetivo del proyecto. En este proceso fueron utilizados los modelos, principios matemáticos $y$ de ingenieria y las ecuaciones que describen y explican el comportamiento de la presión y demás variables involucradas en las pruebas multirata y en el régimen de flujo pseudoestable, para generar las soluciones analiticas basadas en la aplicación de la TDST y con las cuales es posible determinar la presión promedia de yacimiento. La validación de las soluciones que produjo resultados aceptables, permitió concluir que las soluciones son aplicables a casos reales de campo de la industria petrolera.

\section{Solución para Yacimientos Homogéneos}

Cerrados: La presión promedia de yacimiento adimensional en un sistema cerrado esta dada por:

$\frac{d \bar{P}}{d t}=-\frac{q \beta}{A \phi h c_{t}}$

$\bar{P}_{D}=\frac{2 \pi k h}{q \mu \beta}\left[P_{i}-\bar{P}\right]$

En unidades de campo la Ecuación 1.1 quedaria:

$\bar{P}_{D}=\frac{k h}{141.2 q \mu \beta}\left[P_{i}-\bar{P}\right]$

Despejando la presión promedia de Ecuación 1.2:

$\bar{P}=-\frac{q \mu \beta}{2 \pi k h} \bar{P}_{D}+P_{i}$

Por lo tanto:

$d \bar{P}=-\frac{q \mu \beta}{2 \pi k h} d \bar{P}_{D}$

La derivada del tiempo adimensional es:

$d t=\frac{A \phi \mu c_{t}}{k} d t_{D A}$

Substituyendo las Ecuaciones 1.5 y 1.6 en la Ecuación 1.1 y resolviendo se obtiene:

$\overline{P_{D}}\left(t_{D d}\right)=2 \pi t_{D A}$

La presión adimensional durante el estado pseudoestable, en un yacimiento circular cerrado es una función lineal del tiempo adimensional. La ecuación que describe este regimen de flujo es: 
$P_{D}\left(t_{D A}\right)=\overline{P_{D}}\left(t_{D A}\right)+\ln r_{e D}-\frac{3}{4}$

Para tiempos de producción largos, la función de la derivada de presión genera una línea recta de pendiente unitaria y que corresponde al estado pseudoestable:

$\left(t_{D A}^{*} P_{D}^{\prime}\right)=2 \pi t_{D A}$

Reemplazando la Ecuación 1.7 en la Ecuación 1.8 se obtiene:

$P_{D}\left(t_{D A}\right)=2 \pi t_{D A}+\ln r_{e D}-\frac{3}{4}$

Dividiendo la Ecuación 1.10 en la Ecuación 1.9.

$\frac{P_{D}}{\left(t_{D A}^{*} P_{D}^{\prime}\right)}=1+\frac{1}{2 \pi t_{D A}}\left(\ln r_{e D}-\frac{3}{4}\right)$

Substituyendo los términos adimensionales y resolviendo para la presión promedia de yacimiento se obtiene:

$\frac{\Delta P_{q}}{\left(t^{*} \Delta P_{q}^{\prime}\right)}=1+\frac{141.2 q \mu \beta}{k h\left(P_{i}-\bar{P}\right)}\left(\ln \frac{r_{e}}{r_{w}}-\frac{3}{4}\right)$

Finalmente, despejando la presión promedia, se obtiene la ecuación de la presión promedia de yacimiento en pruebas multirata para un pozo localizado en el centro de un yacimiento circular usando los gráficos Log-Log de presión y derivada de presión:

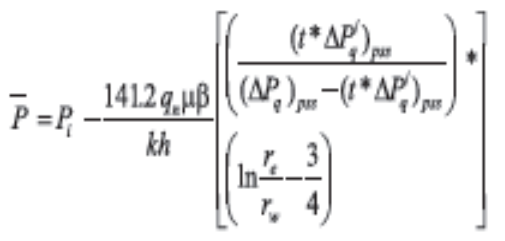

La Figura 1 muestra cada uno de los puntos caracteristicos que son requeridos en la Ecuación 1.13.

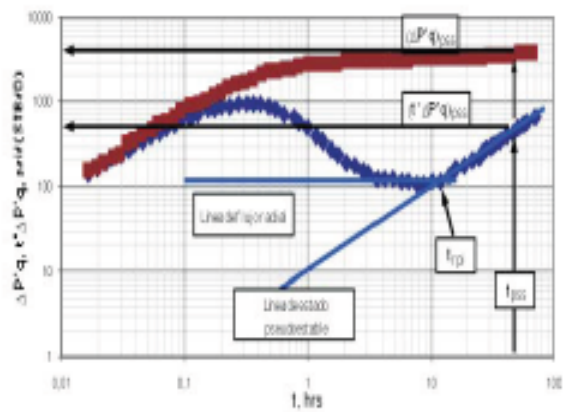

Rgura 1. Esquema ilustrativo de las líneas y puntos caracteristicos requeridos para la determinación de la presión promedia en yacimientos homogéneos cerrados usando la TDST

La permeabilidad de la formación es determinada mediante:

$k=\frac{70.6 \mu \beta}{h\left({ }^{*} \Delta P_{q}^{n}\right)}$

Realizando un procedimiento similar al anterior es obtenida la solución para yacimientos homogéneos cerrados de diferentes geometrias:

$\bar{P}=P_{i}-70.6 \frac{q_{A} \mu \beta}{k h}\left[\begin{array}{l}\left(\frac{\left(t^{*} \Delta P_{q}^{\prime}\right)_{p s}}{\left(\Delta P_{q}\right)_{p s}-\left(t^{*} \Delta P_{q}^{\prime}\right)_{p s}}\right) * \\ \ln \left(\frac{2.2458 A}{C_{A} r_{*}^{2}}\right)\end{array}\right]$

El factor de forma puede ser determinado mediante la siguiente ecuación:

$C_{A}=\frac{2.2458 A}{r_{w}^{2}}\left\{\operatorname{Exp}_{\mathrm{w}}\left[\begin{array}{l}\frac{\pi 0.001055 k t_{\mu \mathrm{s}}}{\phi \mu c_{t} A} \\ \left(\frac{\left(\Delta P_{q}\right)_{m s}}{\left(t^{*} \Delta P_{q}^{\prime}\right)_{p w}}-1\right)\end{array}\right]\right\}^{-1}$

El siguiente procedimiento es sugerido para determinar de la presión promedia en yacimientos homogéneos circulares cerrados, 
aplicando la Tiab's Direct Synthesis Technique (TDST) mediante las soluciones propuestas:

1. Con los datos provenientes de la prueba multirata realizar un gráfico $\log$-Log de presión normalizada $(\Delta \mathrm{Pq})$ y derivada de presión normalizada $\left(t^{\star} \Delta \mathrm{P}^{\prime} \mathrm{q}\right)$ versus el tiempo de prueba, como el que es mostrado en la Figura 1.

2. Identificar las líneas de los regimenes de flujo del yacimiento, la línea horizontal que representa el flujo radial $y$ la línea recta de pendiente unitaria que representa el estado pseudoestable.

3. Determinar la permeabilidad por medio de la Ecuación 1.14, con un valor arbitrario de la derivada de presión de la línea del flujo radial. 4. Con un valor arbitrario de presión normalizada y derivada de presión normalizada de la linea de estado pseudoestable, $(\Delta \mathrm{Pq})$ pss y $\left(t^{*} \Delta P^{\prime} q\right) p s s$, y con la rata de flujo qn y presión de fondo fluyendo Puf correspondientes a ese punto seleccionado, determinar la presión promedia de yacimiento por medio de las Ecuaciones propuestas en este trabajo.

2. Solución para Yacimientos Naturalmente Fracturados en Sistemas Cerrados: La presión adimensional durante el estado pseudoestable, en un yacimiento naturalmente fracturado circular cerrado es una función lineal del tiempo adimensional. La ecuación que describe este régimen de flujo es:

$P_{D}\left(t_{D A}\right)=2 \pi t_{D A}+\ln r_{e D}-\frac{3}{4}+\frac{2(1-\oplus)^{2}}{\lambda r_{e D}^{2}}$

Realizando con la Ecuación 2.1 exactamente los mismos arreglos y reemplazos realizados en el primer caso con la Ecuación 1.8, entonces:

$\bar{P}=P_{i} \frac{141.2 q_{n} \mu \beta}{k h}\left[\begin{array}{l}\left(\frac{\left(t^{*} \Delta P_{q}^{\prime}\right)_{p u}}{\left(\Delta P_{q}\right)_{p u}-\left(t^{*} \Delta P_{q}^{\prime}\right)_{p w}}\right) * \\ \left(\ln \frac{r_{q}}{r_{v}}-\frac{3}{4}+\frac{2 r_{v}^{2}(1-\omega)^{2}}{i r_{\tau}^{2}}\right)\end{array}\right]$
Realizando un procedimiento similar a los anteriores es obtenida la solución para presión promedia en yacimientos naturalmente fracturados cerrados de diferentes geometrias:

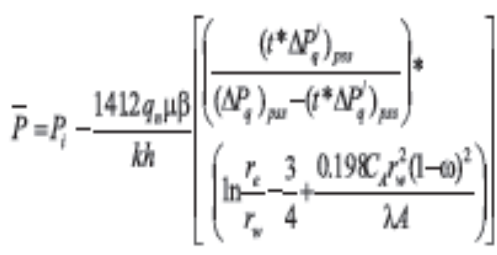

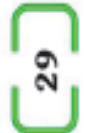

El procedimiento sugerido para determinar la presión promedia en yacimientos naturalmente fracturados, aplicando la Tiab's DirectSynthesis Technique mediante las soluciones propuestas es similar al anterior, con excepción de que en este caso es necesario determinar el coeficiente de almacenamiento adimensional $(\omega)$ y el parámetro de flujo interporoso $(\lambda)$ por medio de:

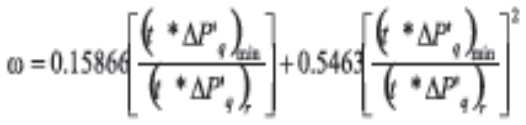

$$
\begin{aligned}
& \lambda=\frac{42.5 h \phi C_{t} u r_{w}^{2}}{B}\left(\frac{t^{*} \Delta P_{Q}^{\prime}}{t}\right)_{\text {mim }}
\end{aligned}
$$

La Figura 2 muestra cada uno de los puntos característicos que son requeridos en la Ecuación 2.2 y 2.3 .

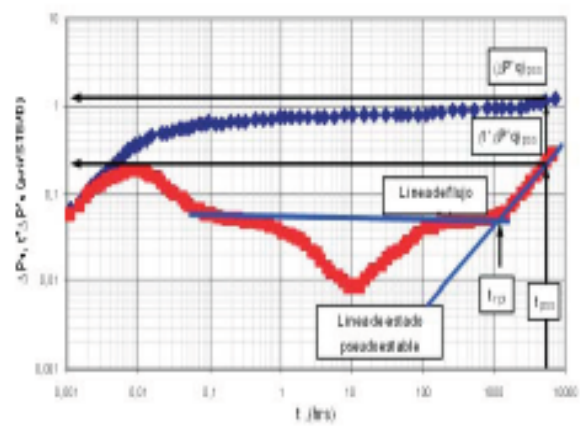

Pgura 2. Esquema listrativo de las lineas y puntos caracteristicos requeridos para la determinación de la presión promedia en yacimientos naturalmente fracturados cerrados usando la TDST 


\section{Ejemplo 1}

Este ejemplo real de campo comprende dos pruebas de presión realizadas a un pozo de un yacimiento circular, una prueba de restauración de presión en la que se determino la presión promedia de yacimiento por medio de los métodos convencionales yuna prueba multirata realizada 8 meses después de la prueba de restauración de presión y en la que se aplico la solución propuesta en este trabajo para determinar la presión promedia y asi poder realizar la validación de la misma. La figura tres muestra los gráficos de presión y derivada de presión de la prueba multirata Los datos de pozo y las propiedades de la rocay el fluido son los siguientes:

$\Phi=13 \%$

$\mathrm{Ct}=1.9 \times 10-51 / \mathrm{psi}$

$\mathrm{h}=80 \mathrm{ft}$

$\mathrm{Pi}=2554 \mathrm{psi}$

$A=62$ acres

$\mu=0.9 \mathrm{cp}$

$\beta=1.3 \mathrm{RB} / \mathrm{STB}$

$\mathrm{rw}=0.33 \mathrm{ft}$

$r e=927.18 \mathrm{ft}$

\section{Solución}

Lapermeabilidaddelaformaciónes determinada por medio de la Ecuación 1.14, con un valor arbitrario de la derivada de presión de la línea de flujo radia de la Figura 3 , por ejemplo $\left(\mathrm{t}^{*} \Delta\right.$
$\left.\mathrm{P}^{\prime} \mathrm{q}\right) \mathrm{r}=0.087 \mathrm{psi} /(\mathrm{STB} / \mathrm{D}):$

$k=\frac{70.6(0.9)(1.3)}{(80)(0.087)}=11.8 m d$

Con un valor arbitrario de presión normalizada y derivada de presión normalizada de la línea de estado pseudoestable, $(\Delta \mathrm{Pq}) \mathrm{pss}$ y $\left(\mathrm{t}^{\star} \Delta\right.$ $\left.P^{\prime} q\right)$ pss, como se muestra en la Figura 3 , con la rata de flujo qn correspondiente a ese punto seleccionado y la $\mathrm{Pi}$, se determina la presión promedia de yacimiento por medio de la solución propuesta para yacimientos homogéneos circulares cerrados propuesta (Ecuación 1.13):

$\left(\Delta P_{q}\right)_{p s s}=10.86 \mathrm{psi} /(S T B / D)$

$\left(f^{*} \Delta P_{q}^{\prime}\right)_{p S O}=0.1648 \mathrm{psi} /(S T B / D)$

$q_{\mathrm{a}}=251 S T B / D$

$\bar{P}=2554-\frac{141.2(251)(0.9)(1.3)}{(11.8)(80)}\left[\begin{array}{l}\left(\frac{(0.1648)}{(10.86)-(0.1648)}\right) * \\ \left(\ln \left(\frac{927.18}{0.33}\right)-\frac{3}{4}\right)\end{array}\right]$

$\bar{P}=2549.1 p s i$

A continuación se muestran los valores de presión promedia de yacimiento obtenidos por diferentes métodos:

\section{Tabla 1. Valores de presión promedia de yacimiento}

\begin{tabular}{|c|c|c|}
\hline Tipo de Prueba de Presión & Método & Presión promedia ,psi \\
\hline Restauración de presión & MBH (Matthews-Brons-Hazebroek) & 2550,3 \\
\hline Restauración de presión & Dietz & 2542,6 \\
\hline Restauración de presión & Ramey-Cobb & 2538,2 \\
\hline Restauración de presión & MDH (Miller-Dyes-Hutchinson) & 2537,3 \\
\hline Restauración de presión & Azari & 2535,4 \\
\hline Prueba Multirata & $\begin{array}{c}\text { Solución propuesta aplicando la } \\
\text { TDST }\end{array}$ & 2549,1 \\
\hline
\end{tabular}






Fgura 3. Valores de presión y derivada de presión para aplicar la solución propuesta mediante la TDST para el ejemplo 1

\section{wO Conclusiones}

- Se desarrollaron diferentes soluciones analíticas para la determinación de la presión promedia de yacimiento en pruebas multirata aplicandola Tiab's Direct Synthesis Technique (TDST), en yacimientos cerrados homogéneos y naturalmente fracturados de diferentes geometras.

- Las soluciones fueron exitosamente validadas a través de los casos reales de campo y los ejemplos simulados, obteniendo valores de presión promedia bastante aceptables comparados con aquellos obtenidos por otros métodos.

- Se logró comprobar que los resultados obtenidos con el tiempo real en el gráfico de la derivada de presión son prácticamente los mismos que los obtenidos utilizando el tiempo equivalente.

- Con estas soluciones propuestas aplicadas a las pruebas multirata, es posible determinar la presión promedia de yacimiento a medida que el pozo continua la producción de fluidos.

- Las pruebas multirata son una herramienta eficaz, para la evaluación de yacimientos (estimación de parámetros). Estas son aplicables para aquellos casos en donde las pruebas típicas, restauración y declinación de presión, presentan complicaciones o no son económicamente viables.

- La Tiab's Direct Synthesis Technique (TDST) es una de las técnicas más prácticas y confiables para analizar datos procedentes de pruebas multirata.

\section{Nomenclatura}

\begin{tabular}{|l|l|}
\hline A & Área de drenaje, ft2 \\
\hline B & Factor volumérico del aceite, RB/STB \\
\hline $\mathrm{Ct}$ & Compresibilidad total, 1/psi \\
\hline $\mathrm{h}$ & Espesor de formación, ft \\
\hline $\mathrm{k}$ & $\begin{array}{l}\text { Permeabilidad horizontal o radial de la } \\
\text { formación, md }\end{array}$ \\
\hline $\mathrm{P}$ & Presión, psi \\
\hline $\mathrm{P}$ & Presión promedia de yacimiento, psi \\
\hline P' & Derivada de presión, psi/hr \\
\hline PD & Presión adimensional \\
\hline P'D & Derivada de presión adimensional \\
\hline Pi & Presión inicial, psi \\
\hline Pwf & Presión del pozo fluyendo, psi \\
\hline $\mathrm{q}$ & $\begin{array}{l}\text { Caudal o rata de flujo del pozo/petróleo, } \\
\text { STB/D }\end{array}$ \\
\hline rD & Radio adimensional \\
\hline re & $\begin{array}{l}\text { Radio de drenaje del pozo, radio externo del } \\
\text { yacimiento, ft }\end{array}$ \\
\hline reD & $\begin{array}{l}\text { Radio adimensional de los limites del } \\
\text { yacimiento }\end{array}$ \\
\hline rw & Radio del pozo, ft \\
\hline $\mathrm{t}$ & Tiempo, hrs, tiempo de prueba \\
\hline $\mathrm{tD}$ & $\begin{array}{l}\text { Tiempo adimensional calculado usando el } \\
\text { radio }\end{array}$ \\
\hline tDA & $\begin{array}{l}\text { Tiempo adimensional basado en el área de } \\
\text { drenaje del yacimiento }\end{array}$ \\
\hline tint & Tiempo de intercepto, hrs \\
\hline tmin & Tiempo mínimo, hrs \\
\hline tpss & Tiempo en el estado psuedoestable, hrs \\
\hline
\end{tabular}




\section{Símbolos griegos}

\begin{tabular}{|l|l|}
\hline$\Delta$ & Cambio, caida \\
\hline$\Delta \mathrm{P}$ & Diferencia de presión, psi \\
\hline$\Delta \mathrm{P}^{\prime}$ & $\begin{array}{l}\text { Cambio de la rata de presión con el } \\
\text { tiempo (derivada de presión), psi }\end{array}$ \\
\hline$\Delta \mathrm{Pq}$ & Presión normalizada, psi/(STB/D) \\
\hline$\Delta \mathrm{Pq}^{\prime}$ & $\begin{array}{l}\text { Derivada de presión normalizada, } \\
\text { psi//STB/D) }\end{array}$ \\
\hline$\lambda$ & Parámetro de flujo interporoso \\
\hline$\Phi$ & Porosidad, fracción \\
\hline$\mu$ & Viscosidad, cp \\
\hline$\omega$ & $\begin{array}{l}\text { Coeficiente de almacenamiento } \\
\text { adimensional o producto } \\
\text { compresibilidad-porosidad de todo el } \\
\text { sistema }\end{array}$ \\
\hline
\end{tabular}

\section{Subíndices}

\begin{tabular}{|l|l|}
\hline$D$ & Cantidad adimensional \\
\hline $\mathrm{f}$ & Formación, fractura, fisura \\
\hline eq & Equivalente \\
\hline $\mathrm{i}$ & Condiciones iniciales o intersección \\
\hline PSS & Flujo en estado pseudo estable \\
\hline $\mathrm{R}, \mathrm{r}$ & Flujo radial, roca \\
\hline usi & $\begin{array}{l}\text { Punto de intersección de la pendiente } \\
\text { unitaria }\end{array}$ \\
\hline
\end{tabular}

\section{" Bíbliografía}

1. DJEBROUNI, A. "Average Reservoir Pressure Determination Using Tiab's Direct Synthesis Technique". Thesis of Master of
Science, University Of Oklahoma, Norman, Oklahoma, 2003.

2. ESCOBAR, F. H. "Análisis Moderno de Presiones de Pozo". Universidad Surcolombiana, Neiva, Noviembre de 2003.

3. MOLINA, M.D. "Determinación de la Presión Promedia en Yacimientos Naturalmente Fracturados, Utilizando la Técnica de Sintesis Directa de Tlab". Tesis de Maestria en Ingenieria de Hidrocarburos. Universidad Industrial de Santander. 2004.

4. MONGI, A. "Application of Tiab's Direct Synthesis Tec hnique to Multi-Rate Flow Tests". Thesis of Masterof Science, University Of Oklahoma, Norman, Oklahoma, 1999.

5. RUSSELL, D. G. "Determination of Formation Characteristics from Two rate Flow Tests". Paper SPE presented at the SPE Fall Meeting held in New Orleans, October 6-9, 1963.

6. TIAB, D., and Boulenouar, D. "MultiRate Testing for Vertical Wells in Naturally Fractured Reservoirs". Paper SPE 88558 presented at the SPE Asia Pacific Oil and Gas Conference and Exhibition held in Perth, Australia, 18-20 October, 2004.

7. TIAB, D., Ispas, I.N., Mongi, A., and Berkat, A. "Interpretation of Multirate Tests by the Pressure Derivative -I. Oil Reservoirs". Paper SPE 53935 presented at the 1999 SPE Latin American and Caribbean Petroleum Engineering Conference held in Caracas, Venezuela, 21-23 April, 1999. 